Córima, Revista de Investigación en Gestión Cultural

ISSN electrónico: 2448-7694

Universidad de Guadalajara

Sistema de Universidad Virtual

México

corima@udgvirtual.udg.mx

\title{
Las fundaciones de arte contemporáneo en Valencia, España: características, perfiles y tipologías
}

\author{
Carmen García Almenar ${ }^{1}$ \\ Universidad Politécnica de Valencia, España
}

DOI: $10.32870 /$ cor.a4n7.7337

[Recibido: 12/12/2018; aceptado para su publicación: 17/05/2019]

\section{Resumen}

El objetivo del artículo es realizar un análisis del trabajo desarrollado por las fundaciones de arte contemporáneo en la ciudad de Valencia, España, para reflexionar sobre su descripción y caracterización como espacios culturales de la ciudad. Para llevar a cabo este análisis se comparan los datos obtenidos de las entrevistas a los directores de las fundaciones de arte contemporáneo de Valencia. El resultado principal fue que las fundaciones, aunque muestran características comunes, presentan perfiles muy particulares. Es importante tener en cuenta que, a pesar de que presentan proyectos culturales consolidados dentro de la oferta cultural de la ciudad, quedan asuntos pendientes para reflexionar, como el de la evaluación de los proyectos llevados a cabo, el mecenazgo, o los espacios para el arte emergente. Con el primer análisis de resultados se puede

\footnotetext{
1 Correo electrónico: aga2047@hotmail.com
}

CÓMO CITAR ESTE ARTÍCULO:

García Almenar, C. (2019). Las fundaciones de arte contemporáneo en Valencia, España: características, perfiles y tipologías. Córima, Revista de Investigación en Gestión Cultural, 4(7). doi: $10.32870 /$ cor.a4n7.7337 
comprobar que las fundaciones de arte contemporáneo realizan una labor social, en relación con el ámbito del arte y la cultura, similar a la función que desempeñan los museos.

\section{Palabras clave}

Colecciones, mecenazgo, arte contemporáneo, evaluación, educación artística, Valencia.

\section{Introducción}

Las fundaciones de arte contemporáneo son organizaciones sin ánimo de lucro que tienen entre sus objetivos proteger, dinamizar y crear arte contemporáneo. Cuando hablamos de arte contemporáneo, nos referimos, fundamentalmente, a las expresiones artísticas que están apareciendo en el presente, al momento histórico en el que la sociedad comparte el mismo contexto con las producciones artísticas y culturales. Tatarkiewicz explica que el arte es una actividad humana consciente, propone las características que distinguen el arte de otras actividades humanas, y, que por su relación con el receptor de la obra, destacan las siguientes:

1) El arte es expresión. Depende de la intención del artista.

2) El arte produce experiencia estética. Se pone atención al efecto que produce en el espectador.

3) El arte produce un choque. También se relaciona con el efecto que produce en el receptor (Tatarkiewicz, 1986, pp. 63-67).

En la actualidad el arte no se valora bajo el concepto de belleza, sino que se evalúa por los numerosos lenguajes visuales, por las herramientas técnicas que lo hacen posible, por la importancia que se le otorga al proceso de creación frente al objeto terminado, por su carácter multidisciplinar $\mathrm{y}$, por supuesto, por su compromiso con el contexto histórico en el que se crea. En muchas ocasiones, debido a la contemporaneidad con el observador, existe una falta de comprensión, esta se adquiere con el paso del tiempo y con la interpretación, desde una perspectiva histórica. El arte también está influenciado por la sociedad de masas del contexto temporal en el que la obra surge. Cabe señalar que, de la misma manera que la sociedad de masas estimula la proliferación del arte, muchas de las obras se pierden, junto con su autor, en la multitud de la sociedad.

Cuando hablamos del arte contemporáneo nos estamos refiriendo a las características centradas en la expresión, en la experiencia estética y en la recepción del espectador. Se considera que estos tres factores hacen del arte contemporáneo una herramienta para reflexionar y criticar la realidad en la que la obra se creó. Este concepto de compromiso se vincula con lo que Janet Wolff 
explica sobre el mensaje de las obras de arte: "lo que el autor o artista dice en la obra es, en realidad (o quizás habría que decir también), la afirmación de un grupo social y su visión del mundo" (Wolff, 1981, p. 143).

Es innegable la capacidad socializadora del arte. Sus mensajes Ilaman la atención, cuestionan, critican y provocan. Esta función socializadora es uno de los aspectos que justifican su presencia en la cotidianidad de la sociedad y los esfuerzos por llevar a cabo su promoción, además de que se considera imprescindible en este trabajo. Como apunta Paloma Rueda, no todo el arte, por el hecho de ser contemporáneo, es válido en el proceso humanizador y en el desarrollo cultural. ${ }^{2}$

Las fundaciones de arte contemporáneo se rigen, a nivel nacional, por la Ley 50/2002, de 26 de diciembre, de fundaciones, según la cual:

1) Son fundaciones las organizaciones constituidas sin fin de lucro que, por voluntad de sus creadores, tienen afectado de modo duradero su patrimonio a la realización de fines de interés general.

2) Las fundaciones se rigen por la voluntad del fundador, por sus Estatutos y, en todo caso, por la Ley.

A pesar de ser organismos privados, las fundaciones tienen que cumplir la promesa de no contar con algún beneficio económico. Realizan su labor por y para la sociedad en la que desarrollan su proyecto cultural. Por esta razón, se pueden considerar "una institucionalización de la sociedad civil" (Jiménez Escobar, 2013, p. 165). Otros autores, como Isabel de la Torre (De la Torre, 2003, p. 122) destacan que "estas empresas u organizaciones se caracterizan por mostrar una cultura altruista, solidaria y cooperativa, desarrollar una actuación propia y diferenciada dirigida al bienestar general y producir bienes que no se pueden incluir en el sector público ni tienen carácter estrictamente privado".

Las competencias de las fundaciones han quedado recogidas en algunos estatutos de autonomía, como en el caso del Estatut d'Autonomia de la comunidad valenciana, en el cual queda proclamada la competencia sobre las fundaciones en el artículo 49 del título IV en el que se expone lo siguiente:

Les Competències:

\section{Article 49}

La Generalitat té competència exclusiva sobre les matèries següents:

23a. Fundacions i associacions de caràcter docent, cultural, artístic i benèfic assistèncial, de voluntariat social i semblants, l'àmbit principal d'actuació de les quals siga la Comunitat Valenciana.

\footnotetext{
2 Paloma Rueda es profesora en el Departamento de Didáctica de la expresión musical, plástica y corporal de la Universidad de Valencia y participó en el grupo de discusión realizado con motivo del proceso de investigación de la tesis La labor social de las fundaciones de arte contemporáneo en la ciudad de Valencia.
} 
A nivel autónomo, en la comunidad valenciana quien regula el marco jurídico de las fundaciones es la Ley 9/2008, de 3 de julio, y se ha desarrollado en el Decreto 68/2011, de 27 de mayo, del Consell. En esta ley, a las fundaciones se les considera como "instrumento privado, surgido en la esfera de la libertad, para cumplir con protección de la administración, fines a los que ésta por sí sola no puede atender y que encajan en el ámbito de la función social de la propiedad."

En este artículo se pretende analizar y describir las fundaciones de arte contemporáneo de la ciudad de Valencia (España), con el objetivo de examinar el papel que desempeñan en la oferta cultural. Otro de los objetivos es reflexionar sobre el trabajo en red para desarrollar proyectos que lleguen a públicos potenciales y consoliden los hábitos culturales de los ciudadanos.

La metodología empleada para el análisis fue la realización de entrevistas personales con los directores de las fundaciones de arte contemporáneo de la ciudad de Valencia. Se excluyeron de este estudio las fundaciones de entidades bancarias y otro tipo de empresas, porque su actividad puede estar vinculada a la mejora de la imagen de la empresa, a conseguir notoriedad, o simplemente a reinvertir el excedente de capital, como en el caso de las cajas de ahorro. Para evitar este conflicto de intereses solo se tomaron en cuenta las fundaciones que se sostienen gracias a la pasión de sus fundadores por el arte.

A través de las entrevistas se recogió información sobre diferentes aspectos que posteriormente se compararon, lo que condujo a una reflexión sobre el trabajo que realizan estos espacios culturales. Los bloques temáticos que se plantearon fueron los siguientes: colección, actividad expositiva, actividades didácticas y de formación, colaboraciones y financiación, mecenazgo, promoción y difusión, mercado del arte y organigrama.

Como señala Olga Martínez, los museos desempeñan la función de difundir sus colecciones y sus actividades, por eso son imprescindibles las exposiciones (Martínez Álvarez, 2002). En el caso de las fundaciones de arte contemporáneo sucede lo mismo. La mayoría desarrollan exposiciones temporales a lo largo del año, esta actividad es la más importante para difundir y promocionar el arte contemporáneo.

Los museos están evolucionando en la manera de presentar sus proyectos, se están convirtiendo en centros vivos de creación y pensamiento (Del Alisal, 2007, p. 230). Pueden encontrarse ciertas similitudes entre las fundaciones estudiadas y los espacios de las instituciones públicas, como el interés por ofrecer una experiencia integral y sensorial que le dé un valor agregado a la exposición, con visitas guiadas teatralizadas, música en directo, conferencias, talleres y actividades de formación y reflexión en torno a las exposiciones.

La conclusión principal giró alrededor de la idea de que todas las fundaciones estudiadas ofrecen perfiles diferentes y orientan su trabajo de acuerdo con su 
visión sobre el arte contemporáneo. Así, mientras algunas presentan programas expositivos de artistas más o menos consolidados, otras ofrecen premios para dar una oportunidad a las nuevas propuestas, o investigan sobre los nuevos espacios para el arte. Por otra parte, es necesario replantearse sistemas de evaluación más operativos y organizados, ya que, en general, se observa una tendencia a limitar los indicadores a solamente realizar el conteo del número de visitantes en los museos.

\section{Las fundaciones analizadas}

Las fundaciones son fruto de la iniciativa particular, son una muestra evidente de los intereses de la sociedad civil, al menos de parte de los individuos que la conforman. Isabel de la Torre explica que el término sociedad civil está relacionado con "el ejercicio de una ciudadanía que defiende intereses colectivos en espacios públicos". Y añade que su función es "reclamar la atención de cualquier forma de poder que opera en espacios públicos, para resolver demandas insatisfechas o anticipar la protección de nuevas necesidades de grupos sociales" (De la Torre, 2003, p. 120).

De acuerdo con lo anterior, la fundación es una herramienta de expresión y de participación que visibiliza y valora las iniciativas de la ciudadanía, es importante que las instituciones las tengan en cuenta en sus propuestas culturales, para intentar atender la diversidad de la sociedad civil. En este sentido, José Luis Pérez Pont, director del consorcio de museos de la comunidad valenciana, señala lo siguiente:

es interesante la incorporación de esas fundaciones al panorama cultural de una ciudad, una región o un entorno porque de algún modo aportan una diversidad en la oferta cultural". Y añade que es "es una acción positiva en cuanto a que atrae fondos privados para desarrollar una acción cultural que tiene una repercusión pública. Es un aspecto positivo que viene a sumar y añade valor a la oferta cultural de la ciudad. ${ }^{3}$

El perfil de fundación de arte contemporáneo que no está vinculada a ninguna empresa y a sus actividades de patrocinio, lo presentan, en la ciudad de Valencia, nueve fundaciones, de las cuales ocho han sido entrevistadas. Además, se incluye la colección Martínez Guerricabeitia porque, a pesar de no estar inscrita como fundación, tiene el mismo origen que la mayoría de ellas, con la diferencia de que fue donada a la Universidad de Valencia, encargada de su gestión a través de la FGUV.

\footnotetext{
3 Extracto de la entrevista realizada a José Luis Pérez Pont el 15 de enero de 2019 incluida en el desarrollo de la Tesis La labor social de las Fundaciones de Arte Contemporáneo de la ciudad de Valencia.
} 
Las fundaciones entrevistadas fueron las siguientes: la Fundación Chirivella Soriano, cuyo representante fue José Luis Giner, responsable del departamento de exposiciones; la Fundación Per Amor a l'Art, representada por Nuria Enguita, directora del centro de arte del que disponen; la Fundación Mainel, cuya entrevista se realizó a Jorge Sebastián, uno de los patronos y responsable del departamento de innovación e investigación; la Fundación La Posta, Guillem Cervera, su fundador, fue quien respondió a la entrevista; la Fundación Cañada Blanch, a través de Paula Sánchez, directora del departamento de gestión; la Fundación Anzo, donde se entrevistó a Amparo Iranzo, fundadora; la Fundación Josep Renau, cuya entrevista se realizó a su director Emili Payà y la Fundación Hortensia Herrero, cuyo representante fue Fernando Jiménez, responsable de proyectos.

Como se indicó en líneas anteriores, se excluyeron las fundaciones pertenecientes a entidades bancarias, en este caso fueron la Fundación Bancaja y la Fundación Caja Mediterráneo. Tampoco se incluyó la Fundación Antonia Mir porque a pesar de estar inscrita en la ciudad de Valencia, tiene su sede en otra localidad. Por último, se excluyó la Fundación del Palau de les Arts Reina Sofia porque es de carácter público y no es iniciativa de la sociedad civil, además de dedicar su actividad únicamente a las artes escénicas.

Una de las características que comparten algunas de las fundaciones es que con frecuencia desarrollan proyectos simultáneos que, como sostienen algunos autores como Pau Rausell, compensan el riesgo que pueden suponer (Rausell, 2012, pp. 66-67). Por ejemplo, vemos cómo la Fundación Mainel desarrolla dos líneas de trabajo, una en relación con el arte y la cultura, y la otra relacionada con la cooperación para el desarrollo. O la Fundación Cañada Blanch, que desarrolla un proyecto cultural en paralelo con otro orientado a la educación y la formación, relacionado con estudios económicos. Incluso la Fundación Per Amor al Art, que lleva a cabo un proyecto de apoyo a la investigación de enfermedades poco comunes y otra vía dirigida hacia el arte contemporáneo, su promoción y la sensibilización de los usuarios.

Otra característica que presentan la fundaciones que disponen de sede es que han contribuido a la restauración de edificios que posteriormente se han puesto al servicio de la ciudadanía. Esto se observa con claridad en la Fundación Chirivella Soriano, quienes adquirieron un antiguo palacio gótico para restaurarlo, - la FPAA, que ha restaurado las instalaciones de una antigua fábrica de bombas hidráulicas, una bodega del siglo XV y un refugio de la guerra civil, todo esto se incluyó en sus instalaciones y sus espacios de visita.

Esta situación posibilita que la inversión pública en cultura se complemente con las inversiones privadas, lo que supone el fortalecimiento del sector cultural; además, en este tipo de redes encontraría una forma de aumentar su impacto en la sociedad. Algunos autores aseguran que "las organizaciones culturales 
contribuyen a la promoción de la identidad territorial y refuerzan la memoria histórica local al hacerse cargo de la gestión de espacios como palacios o antiguas fábricas" (Rausell, 2012, p. 58).

\section{La colección}

La colección es el punto de partida del análisis de las fundaciones de arte contemporáneo, porque el coleccionismo ha sido, a lo largo de la historia, un factor importante en la configuración del gusto (Del Alisal, 2007, p. 226). En el momento que alguien reúne un conjunto de obras en torno a un discurso o con unas características determinadas, las dota de cierta distinción sobre el resto de los objetos. Además, la colección hace que el coleccionista destaque en la sociedad como alguien con posibilidades de recrearla. Es una puesta en valor recíproca.

Existe una definición del término colección museográfica en la Orden de 1991, de la Conselleria de Cultura, Educación y Ciencia, según la cual las colecciones reunirán tres requisitos: disponer de instalaciones adecuadas, presentar un inventario y ser visitables al público (Orden del 6 de febrero de 1991). Sin embargo, debido a la complejidad de los escenarios actuales, el desarrollo de las nuevas tecnologías y los nuevos espacios expositivos, como las redes sociales, está cambiando la experiencia estética y el concepto de colección debe adaptarse a las nuevas necesidades.

Las colecciones representan un gran potencial para favorecer el desarrollo cultural de la sociedad a través del conocimiento y la crítica. Por esta razón no pueden quedar fuera de los proyectos del ámbito de la investigación en torno a los nuevos espacios para el arte y las nuevas maneras de experimentarlo.

Por último, es necesario valorar las colecciones privadas que se abren al público por varios motivos, según Del Alisal (2007, p. 228).

- Al hacerse públicas influyen en el gusto de quien las disfruta.

- Complementan las colecciones públicas y fortalecen la oferta cultural de la ciudad.

- $\quad$ Son reflejo de los intereses de los ciudadanos que las crean, mostrando con ellas la diversidad de la sociedad civil.

Con esta breve aproximación a la definición del término colección, en el mapa aparecen otros tipos de colecciones: las de intangibles. Este concepto no es nuevo puesto que en la ciudad ya existe un museo sin colección, conocido como el museo de las ideas, el MUVIM. Sin embargo, todavía puede sonar raro que una colección no esté formada por obras físicas. 
Según Martínez Álvarez, (2002, p. 272) quien cita a Prats (1999) "una colección es digna de un museo, no por el tema que trata, sino por su coherencia, completitud, documentación y estado de conservación de los objetos de la colección". Con esta definición se ha intentado revisar hasta qué punto las colecciones de las fundaciones de arte contemporáneo estudiadas son dignas de mención.

Las entrevistas realizadas a las fundaciones abrían con una pregunta sobre su colección, todos los entrevistados respondieron de acuerdo con la definición tradicional de lo que debe ser una colección. Todas disponen de obras, de mayor o menor importancia y en mayor o menor volumen, excepto la Fundación La Posta que, a pesar de no disponer de fondos, realiza una labor cultural muy dinámica en torno al hecho artístico y la cultura visual, explora nuevos recursos de contemplación en continua reflexión con el contexto social, por este motivo se refieren a la fundación como centro de investigación.

Ante la pregunta relativa a la existencia de una colección, Guillem Cervera respondió: "Nuestra actividad, cuando hicimos el centro de investigación, no la basamos en torno a una colección, a partir de la cual se montan exposiciones, una colección que se revisa y que es el objeto de estudio. Nosotros estamos volcados en las actividades docentes y de participación".

Podemos concluir que está contribuyendo a la generación de patrimonio contemporáneo. Producen ideas de las que pueden nacer obras materializadas o sentimientos sensibles a las expresiones culturales contemporáneas. Estos hechos son imprescindibles para la aceptación de lo que comúnmente se entiende por obra de arte contemporáneo.

A partir del análisis de las fundaciones de arte contemporáneo en la ciudad de Valencia se supo que todas han nacido del interés personal de sus fundadores por el arte. Por su interés y gusto personal, ellos iniciaron sus colecciones privadas, las cuales crecieron hasta que fue difícil almacenarlas. Uno de los coleccionistas señala que además del problema de almacenamiento y conservación, se plantearon la necesidad de abrir su colección al público cuando se dieron cuenta de que la sociedad tiene que conocerlas. Es decir, consideran que el arte, de alguna manera, pertenece a la sociedad. No hay que entender aquí que renuncian a su titularidad como coleccionistas, sino que valoran la función social del arte y deciden compartirla con el público.

Otra característica que tienen en común las fundaciones analizadas es que las obras que completan su colección son de arte contemporáneo, tienen piezas de la segunda mitad del siglo XX hasta la actualidad. Dentro de ese periodo cada una se ha orientado en una dirección específica, con temáticas y técnicas diferentes. Podemos encontrar colecciones en las que predomina la pintura o la fotografía, y otras en las que los medios técnicos son diferentes. También hay colecciones en las que destacan autores reconocidos y otras en las que, además del autor, se busca 
una temática concreta, como el caso de la colección Martínez Guerricabeitia, que se nutre de obras con un importante carácter de denuncia social.

En esta variedad de perfiles hay una colección que destaca por su carácter variopinto. Su peculiaridad reside en la manera en la que se conformó. Nos referimos a la colección de la Fundación Mainel. Este conjunto de obras se ha confeccionado por diferentes vías. Por un lado, están las pinturas que reunieron sus fundadores, don José Rodrigo y doña Carmen Orts, las cuales pertenecen a la segunda mitad del siglo XX. Por otra parte, hay un conjunto de obras que se quedan como depósito de las que no se han vendido en las subastas benéficas que realiza la fundación. Estas obras son cedidas por artistas para colaborar en estas actividades de recaudación. La colección está formada por los ganadores del Premio Nacional de Pintura que se organiza cada año. Hay un jurado experto que decide la obras que pasarán a formar parte de los fondos de la colección.

Hay que destacar lo peculiar de la colección Cañada-Blanch porque, comparada con la de sus compañeras, es de reciente creación y no muestra un hilo conductor claro. Mantiene cierta similitud con una de las líneas de Mainel, puesto que también se integró a partir de una serie de convocatorias realizadas en los años noventa. Actualmente están analizando sus fondos para construir un hilo argumental y dotarla de más sentido. Además, se plantea la ampliación de la colección con fotografías adquiridas en el premio Photon, esta característica también la comparte con Mainel.

Creemos conveniente destacar la colección de la FPAA porque es la única que se ha consolidado con el asesoramiento de un experto, Vicente Todolí, el cual participa en proyectos de este tipo cuando uno de los objetivos es la apertura al público. Este podría ser un ejemplo de coleccionista que no busca un rendimiento mercantil de su inversión, sino una promoción del arte.

A partir de este primer análisis destaca el papel que desempeñan las fundaciones de arte contemporáneo respecto al patrimonio actual, sobre todo si tenemos en cuenta la definición de cultura, propuesta por Olaia Fontal. "La cultura hace referencia a todos los ámbitos que contribuyen a caracterizar un contexto, en un tiempo determinado" (Fontal, 2006, p. 11). En este sentido, el arte actual puede considerarse una clave cultural del presente. Esta relación con el presente es la que consideramos importante para reclamar la atención de los ciudadanos. Es necesario que se entienda el valor del arte actual como parte del patrimonio que, como en este caso, todavía carece del valor que le otorga el paso del tiempo, reside en el vínculo que se establece con el contexto en el que se crea y con los ciudadanos que lo viven (Gómez Redondo, 2011, p. 110). 


\section{Actividad expositiva y actividades didácticas}

Para seguir el análisis del trabajo que realizan estas instituciones privadas revisaremos la manera en que llevan a cabo su programa expositivo. Generalmente todas muestran una serie de exposiciones temporales anuales, las cuales se organizan a partir de las obras que son propiedad de la fundación. En caso de que el hilo argumental requiera de alguna otra obra externa, se realizan los trámites necesarios para completar la muestra. De nuevo, la Fundación Mainel y la Fundación Cañada-Blanch se salen del guion. Mainel solo realiza una exposición al año, la que muestra las obras seleccionadas en el premio nacional que organizan. Y Cañada-Blanch, que participa en la exposición de fotografía del festival Photon, del que principales son patrocinadores.

Junto con las exposiciones temporales aparecen las visitas guiadas. Todas las fundaciones de este estudio, excepto la Fundación Mainel, ofrecen esta actividad, pero se diferencian en el modo de llevarlas a cabo. De acuerdo con las características de cada uno de estos espacios y los recursos disponibles, encontraremos diferentes planteamientos. Por una parte, existen visitas guiadas para grupos, previa cita. Con reservación también pueden realizarse visitas guiadas sin que haya mínimo de participantes. Estas visitas, en algunos casos, son dinamizadas; según los responsables de los espacios, consisten en darle un valor añadido con "teatralizaciones" que convierten la visita de contemplación pasiva en una experiencia susceptible de permanecer en la memoria.

En la oferta didáctica pueden distinguirse dos líneas de trabajo: las actividades didácticas en torno a las exposiciones y las actividades de formación que se desarrollan en el tiempo. Estas se han convertido en una práctica habitual en los museos, no solo para escolares, sino también para adultos y grupos familiares. Estos talleres, que favorecen la comprensión de las obras, también pueden encontrarse en las fundaciones; sin embargo, son diferentes.

Para las fundaciones es difícil desarrollar una programación didáctica estable. Solo la FPAA tiene la capacidad de ofrecer un proyecto educativo con continuidad, la cual programa, a través del Centro de Arte Bombas Gens, toda una serie de actividades en torno a su colección. Esta fundación distingue entre el programa de actividades didácticas y el programa educativo. Nuria Enguita lo explica de esta manera en la entrevista realizada:

Educación tiene que ver no tanto solo con las actividades escolares o para institutos sino una especie de formación no reglada que tiene que ver con actividades que ocurren en el tiempo, que son procesos formativos, porque se establecen con una continuidad. Ese es el criterio que distingue nuestras actividades. Fue muy difícil definirlo y al final decidimos que, por ejemplo, una conferencia, que empieza y termina, es una actividad. $Y$ en educación están más actividades como el grupo "En marcha", que es un grupo que tenemos con los vecinos de Marxalenes. Son personas adultas, jóvenes o mayores, pero no 
están vinculados a ningún colegio o instituto. Durará de 6 meses a un año. En ese momento habrá unos resultados, eso es lo que se ha entendido como educación.

De las dificultades que presentan el resto de fundaciones para ofrecer este tipo de proyectos, destacan la falta de espacio para desarrollarlos, no contemplarlos en la misión de su organización o la falta de recursos humanos. En ocasiones, al colaborar con otros agentes culturales plantean talleres, como sucede en la Fundación Chirivella Soriano al colaborar con World Press Photo. En este caso, la Fundación World Press Photo se encarga del taller didáctico basado en la exposición de fotografía que la fundación acoge en su sede.

Las fundaciones Anzo y Josep Renau, a pesar de no disponer de infraestructura ni personal para la realización de actividades didácticas, desarrollaban talleres didácticos con su colección, pues otros organismos acogieron sus obras en otros espacios, como el IVAM.

Llama la atención el caso de la Fundación La Posta, pues aunque cuenta con uno de los espacios más reducidos, desarrolla diferentes ciclos de audiovisuales y talleres de cine, surgidos de sus procesos de investigación. En este caso, el público debe ser adulto y con conocimientos del tema. También plantean, en colaboración con otros colectivos, exposiciones para estudiantes.

Por último, hay fundaciones donde no se realiza este tipo de proyectos, como sucede con la Fundación Mainel y la Cañada Blanch. En el primer caso, aunque reconocen que sería interesante realizarlos, señalan la dificultad de diseñar un taller en torno a una exposición en el que las obras son tan dispares como los artistas que se postulan para la convocatoria del premio. Lo que sí plantean son ciclos de conferencias sobre cultura visual contemporánea en los que participan expertos en la materia; además, se invita a reflexionar en torno a los asuntos relacionados con el arte y la cultura.

Debido a que la Fundación Cañada Blanch no dispone de una colección consolidada, no se han planteado este tipo de proyectos didácticos, aunque reconocen que en la medida en que la colección se afiance, realizarán alguna propuesta.

Estas actividades didácticas y de formación son una pieza importante dentro de la oferta cultural de la ciudad, porque promueven la reflexión y el entendimiento de las exposiciones, y facilitan el diálogo entre el público y el arte contemporáneo.

\section{Mecenazgo}

El mecenazgo es, como apunta Manuel Palencia-Lefler, "un conjunto de actuaciones de interés social que hace una empresa o una persona, sin una pretensión directa en la obtención de un beneficio propio en términos de imagen o de aceptación 
pública" (Palencia-Lefler Ors, 2007, p. 158). Sin embargo, Palencia-Lefler señala que otros autores como Parés (1994) argumentan que todo acto de mecenazgo conlleva una contrapartida. Esta contrapartida puede ser de tipo económico, en forma de desgravación, de reconocimiento, o de una mejora de imagen y de prestigio. El tema del mecenazgo se estudia, por una parte, debido a que la fundación, como institución, podría ofrecerles a los artistas la oportunidad de dar a conocer su obra y, por otra, porque la fundación puede beneficiarse de donaciones de entidades o personas físicas que también se consideran sus mecenas.

Para fomentar el mecenazgo la comunidad valenciana puso en marcha la Ley 9/2014, de 29 de diciembre, de impulso de la actividad y del mecenazgo cultural en la Comunitat Valenciana. En vista de la poca repercusión que tuvo, se ha planteado su modificación, por tanto, se aprobó la Ley 20/2018, de 25 de julio, de la Generalitat, del mecenazgo cultural, del científico y del deportivo no profesional, en la Comunitat Valenciana.

Este asunto es delicado porque deberíamos preguntarnos quién tiene la responsabilidad de ofrecer espacios para el arte. Un artista consolidado, con cierta trayectoria dentro del mundo del arte y activo en este mercado puede acceder al museo como institución, pero hasta llegar a sus salas ha recorrido un camino de premios, becas y espacios de arte que no siempre han pertenecido a la institución pública. Las escuelas de arte y la universidad deberían pensar en fórmulas para diseñar los caminos que el artista necesita recorrer. Podemos plantear la posibilidad de gestionar espacios experimentales ofrecidos por organismos públicos locales para abarcar la demanda de arte emergente en una ciudad. Junto a estos espacios, conviven las salas privadas que en ocasiones abren convocatorias para otorgar premios a los artistas jóvenes, o abren convocatorias para ofrecer sus salas a las nuevas propuestas; sin embargo, no hay que olvidar que son organismos privados que están arriesgando sus recursos al apostar por este tipo de proyectos.

Ninguna de las fundaciones estudiadas propone un plan de mecenazgo estable. Algunas apuestan por trabajos que consideran que encajan con su proyecto expositivo y ofrecen sus salas, incluso colaboran en la producción de la obra, pero siempre de manera limitada. En el caso de Chirivella Soriano está la convocatoria Espai d'Arcs, para los estudiantes del último curso del Máster de Producción Artística, con esta oportunidad tienen un escaparate para mostrar su obra en alguno de los espacios de arte contemporáneo de la ciudad.

Una iniciativa similar es la que llevan a cabo en la Fundación Hortensia Herrero, ellos colaboran como patrocinadores, con el Centre del Carmen - Consorci de Museus de la Comunitat Valenciana y la Facultad de Bellas Artes de la UPV en el PAM! PAM! (Muestra de Producciones Artísticas y Multimedia). 
Aunque no se ofrece ningún plan de mecenazgo, brindar la posibilidad de acceder a un premio, como lo hacen Mainel y Cañada-Blanch, es una gran oportunidad en la carrera de los artistas emergentes. Además, todas las fundaciones ofrecen estrategias de difusión del arte, esto da a conocer a los artistas y a su obra. Todas las fundaciones utilizan las NTIC para desarrollar sus planes de difusión, especialmente en las redes sociales que llegan a un público amplio y diverso de forma rápida y eficaz. Aunque algunas de las fundaciones no mantienen su página web actualizada, internet está ganando terreno como espacio de publicación de los trabajos culturales, no solo a nivel informativo, sino también como espacio expositivo.

Para cerrar este apartado podemos decir, de acuerdo con la terminología utilizada por Umberto Eco (1995) en su obra Apocalípticos e integrados, que las fundaciones estudiadas contribuyen a la democratización de la cultura, abren sus puertas y se ponen a la disposición del público de la ciudad y, además, posibilitan el progreso de la democracia de la cultura en la ciudad, debido a que contribuyen al desarrollo del ser humano como agente activo en el ámbito artístico y cultural.

\section{Evaluación}

La evaluación es un proceso con el cual se valora el desarrollo y el resultado de una actividad o proyecto. Se atiende al cumplimiento de los objetivos establecidos, a la definición del proyecto y al correcto desarrollo de la actividad (Roselló Cerezuela, 2008, p. 205).

La evaluación de un proyecto cultural es importante porque entrega datos sobre las necesidades del entorno donde se trabaja y sobre las aportaciones del proyecto que se desarrolle. A partir del análisis de los datos que ofrece la evaluación se revisan aspectos sobre los recursos utilizados, las propuestas llevadas a cabo o la justificación de la inversión de dinero público en un sector de carácter privado.

Como explica Luis Fernando Aguado, desde los años setenta y ochenta del siglo XX fueron muy famosos los estudios sobre el impacto económico de la cultura para justificar la financiación pública de los proyectos culturales (Aguado Quintero, 2010, p. 110). Por tanto, se considera necesario analizar el trabajo de las fundaciones y su manera de evaluar los resultados obtenidos en sus actividades expositivas y didácticas.

Hay dos tipos de evaluación: la externa y la interna. La externa es obligatoria porque la exige el protectorado para asegurar que las fundaciones respeten los principios de transparencia en su gestión. Esta evaluación es anual y cuantitativa, revisa los gastos e ingresos de las fundaciones, además examina que las actividades que se realizan respetan la misión de la fundación. 
La evaluación interna la realiza la fundación para analizar las estadísticas en relación con los parámetros que modifican el proyecto y lo mejoran. No solo se valora, por ejemplo, el número de visitas, sino que se revisan los factores que influyeron en el alta o la baja de visitantes y de qué manera atraer más público en caso de que los números hayan sido bajos. Debe atenderse el diseño del proyecto y el desarrollo de sus distintas fases, localizar los puntos débiles y las fortalezas para avanzar hacia un mayor impacto.

Todas las fundaciones realizan algún tipo de estudio, principalmente contabilizan el número de visitantes. Además, algunas de ellas realizan otro tipo de evaluación, a nivel cualitativo, con informes curatoriales en los que se evalúa el tipo de público, aparición en medios, fidelidad o grado de satisfacción de los asistentes, como sucede en la FPAA, en La Posta o en la CMG. Estos informes son un documento importante porque les ofrece una visión del impacto de sus proyectos, de sus aciertos y de los aspectos que tienen que trabajar para mejorar.

En la evaluación interna hay que hacer un balance social debido a que las fundaciones presentan, por definición, una misión de interés general de la que se espera una aportación a la comunidad.

Según Cabra de Luna (2001, p. 56) el balance social "es un instrumento de información a la entidad para su propia organización, que incluye el mayor número posible de datos de tipo social que juegan un papel importante en el éxito o fracaso de su actividad y de su grado de comunicación con los agentes sociales a través de los que se relaciona".

Como señala de Luna, el balance social no tiene como objetivo reducir a cifras económicas los resultados obtenidos, sino mostrar el impacto que tienen las actividades en la sociedad. Lo plantea como un informe que se presenta cada tres o cinco años para ver la evolución de los datos obtenidos anualmente y, en el caso de las fundaciones, en las memorias y planes de actuación (Cabra de Luna, 2001, p. 69).

\section{Financiación}

La financiación de las fundaciones es uno de los temas importantes. Por definición, ninguna de ellas obtiene beneficio económico derivado de su actividad cultural. Todas ellas se autofinancian a partir de sus recursos e inversiones. En ocasiones reciben ayuda económica a partir de concurso público, pero puede considerarse un ingreso anecdótico. Hay fundaciones con presupuestos anuales muy modestos, como el caso de Mainel (32 $44247 €$ ) o La Posta (36 $78600 €$ ), y otros presupuestos más holgados como el de FPAA o Cañada-Blanch (400 $000 €$ ). Estos presupuestos se reparten entre la organización de exposiciones y otras actividades, el reparto está condicionado por la filosofía de trabajo de cada fundación. 
Existen fundaciones que con menos presupuesto se esfuerzan en ofrecer una programación expositiva potente, mientras que otros presupuestos mayores se reparten y se diversifican entre más actividades. Por ejemplo, en el caso de la Fundación Josep Renau, que no dispone de ningún presupuesto para desarrollar su actividad, su operación principal se basa, por una parte, en las exposiciones y actividades organizadas por el Instituto Valenciano de Arte Moderno (IVAM) porque esta institución pública es la depositaria de la colección Renau. (De hecho, este depósito fue una de las vías de formación de la colección del IVAM y su posterior creación como instituto de arte moderno). Por otra parte, la Fundación Josep Renau participa en la organización de otras exposiciones en colaboración con ayuntamientos.

Por lo general, todas las fundaciones muestran interés por mantener su autonomía en el desarrollo de sus proyectos, la autonomía es posible siempre que los ingresos provengan de fondos propios. En el momento en el que se aceptan aportaciones externas de otras organizaciones o de la instituciones públicas, se corre el riesgo de perder libertad en la organización de sus propuestas. El arte y la cultura pueden ver amenazada su libertad de expresión cuando en el juego intervienen otros actores con intereses diferentes, como pueden ser de carácter político o publicitario.

Por eso las colaboraciones son un terreno delicado que se mueve para buscar puntos en común. Recibir una aportación económica por parte de una empresa interesada en reforzar su responsabilidad social corporativa puede ser un apoyo de gran trascendencia a la hora de organizar una actividad cultural. Pero hay que encontrar intereses comunes en esta relación. Si la empresa ya cuenta con su colección y su fundación, es difícil que acceda a subvencionar un producto cultural que ya ofrece. Sin embargo, sería interesante establecer relaciones de intereses encontrados en los que, por una parte, la empresa privada realice su aportación $y$, por consiguiente, obtenga mayor visibilidad en la sociedad $y$, por otra, que la fundación consiga llevar a cabo un proyecto que, sin esta financiación, sería imposible desarrollar.

\section{Otras características}

Lo que caracteriza a las fundaciones analizadas es que son de tamaño reducido debido al número de trabajadores en su plantilla, en ocasiones inferior a cinco personas, excepto la FPAA que, en su línea relacionada con el arte contemporáneo, correspondiente al Centro de Arte Bombas Gens, cuenta con 20 personas organizadas en diferentes departamentos. Sin embargo, el patronato suele ser relativamente numeroso si se compara con el número de trabajadores. De este depende la aprobación de los proyectos. 
En lo que corresponde a las características físicas o logísticas de las fundaciones, la mayoría de ellas dispone de un espacio reducido para desarrollar su actividad, o en algunos casos ni siquiera existe, lo cual influye directamente en sus propuestas y en las colaboraciones que tienen que establecer para conseguir espacios expositivos. Aunque también existen contrastes si comparamos los 4500 metros cuadrados expositivos de la FPAA con los 50 que tiene disponible la Fundación La Posta.

Llama la atención que la mayoría de las fundaciones se encuentran en el centro histórico-cultural de la ciudad, excepto la fundación Anzo. Esto es una ventaja si pensamos en la posibilidad de establecer un distrito cultural; sin embargo, la periferia queda desatendida, las fundaciones que no se encuentran en el radio de influencia del centro cultural son menos visibles y, por lo tanto, es más difícil su incursión en el tejido artístico de la ciudad.

De acuerdo con la presencia geográfica de los espacios analizados, incluso del conjunto de espacios culturales, se podría trabajar para abrir el centro a la periferia. Chema Segovia, Ramón Marrades, Pau Rausell y Raúl Abeledo citan en su obra un proyecto llamado "Antimuseo". Este es un ejemplo de cómo, desde la periferia, se puede trabajar el espacio público más allá del centro institucional, para crear un espacio de reflexión que acerque la ciudad y el arte a zonas que, por su situación geográfica y social, quedan atrapadas en la marginalidad (Segovia et al., 2015, p. 20).

Uno de los rasgos que favorece la visibilidad de las colecciones para el público es la facilidad de acceso en planta baja. Este es el caso de la FPAA, Chirivella Soriano y La Posta. Sin embargo, Mainel, Cañada-Blanch y la CMG tienen sus salas en la primera planta. Cañada-Blanch, en realidad, utiliza el espacio de La Nau cuando realiza su trabajo en el festival Photon, por lo tanto, la ubicación de su sede no es un inconveniente a la accesibilidad. La CMG se ubica dentro de las instalaciones del edificio de la Nau, a pesar de estar en la primera planta y que esto supone una pérdida potencial de visitantes, por lo que su actividad no pasa desapercibida.

Los horarios también son muy dispares, algunos son muy semejantes a los que ofrece cualquier museo público, en los que se abre todos los días de la semana, excepto el lunes o el martes. Hay otros más reducidos que se adaptan a la exposición que desarrollan, como los de la Fundación La Posta.

Otra de las características que deben señalarse es el precio de la entrada. Todas las fundaciones, excepto Chirivella Soriano, ofrecen acceso gratuito cualquier día de la semana. Pese a lo que se podría suponer, el precio de entrada no es un obstáculo en el consumo de este tipo de producto cultural. Según las estadísticas oficiales, en la mayor parte de las visitas a museos en 2016 se pagó la entrada completa. 
Por último, en las entrevistas realizadas a los responsables de las fundaciones, se les preguntó por el personal que trabaja en el proyecto y la estructura del área de recursos humanos. Se pretende conocer el grado de profesionalización de las fundaciones porque se presupone que si han recibido una formación relacionada con el arte y la cultura es más probable que ofrezcan un producto cultural de calidad.

En general, no cuentan con grupos de trabajo amplios. El caso más numeroso es el de la FPAA, que ronda las 20 personas en la plantilla, organizadas en departamentos. El menos numeroso sería el de la CMG, que desarrolla su proyecto a partir del trabajo de dos profesionales. En la mayoría de las fundaciones el perfil profesional se ajusta a las licenciaturas relacionadas con la historia del arte y las humanidades. En ocasiones se incluyen los estudios en bellas artes, principalmente los que tienen que ver con el desarrollo de las actividades didácticas. Lo que no se menciona son los estudios en gestión cultural, porque se da por hecho que es la experiencia la que se encarga de completar la formación artística. En el caso de Cañada-Blanch sí se menciona el interés por introducir la figura del gestor cultural porque quieren dotar de especificidad al proyecto $y$ consideran que este perfil profesional podría ayudar en el diseño de una propuesta cultural coherente.

Las tareas administrativas generalmente se dejan en manos de personal especializado externo a la fundación. Como ocurre también con los trabajadores que se contratan para el montaje de las exposiciones, el diseño del catálogo y tareas concretas de mantenimiento.

\section{Conclusiones}

A partir del análisis de las entrevistas realizadas a los representantes de las fundaciones de arte contemporáneo de la ciudad de Valencia, se propone una clasificación tipológica. Tomamos como referencia los ejes funcionales de la fórmula MUVIM para establecer las tipologías de las fundaciones de arte contemporáneo que encontramos en la ciudad de Valencia.

1) Fundación expositiva. Predomina la programación de exposiciones en torno a las cuales se organizan otras actividades, aunque estas quedan en un segundo lugar. Generalmente se basan en artistas que forman parte de su colección, de esta manera pueden promocionarla, pero también organizan exposiciones con obras de otros artistas que complementan el discurso de sus fondos o diseñan exposiciones que mantienen su línea de trabajo sin pertenecer a su colección. Este es el caso de la Fundación Chirivella Soriano. 
2) Fundación investigadora. No necesariamente disponen de una colección, pero destaca por ser un centro de investigación de los nuevos espacios para el arte y su relación con el público. De este tipo encontramos la Fundación La Posta, su programación se realiza de acuerdo con el estudio sobre las nuevas formas de contemplación, influenciadas por el auge de las nuevas tecnologías y los recursos digitales.

3) Fundación educadora. Ofrecen un programa de actividades educativas y de formación, relacionadas con los discursos expositivos. Trabajan la sensibilización de los ciudadanos y su participación activa. Estas actividades pueden plantearse a modo de talleres o de jornadas que se prolongan a lo largo de un periodo establecido, su campo de acción es el fomento de la cultura artística y la atracción de públicos. En este grupo encontramos la FPAA y la CMG. Ambas presentan una programación expositiva (estas podrían incluirse en el primer grupo) que se complementa con visitas guiadas, seminarios y jornadas de formación.

4) Fundación difusora. Llevan a cabo un proyecto de edición y transmisión de conocimiento a través de catálogos y publicaciones, ya sea en soporte tradicional o en digital, también realizan conferencias y encuentros con profesionales del mundo artístico. En este grupo se incluyen las que dirigen su actividad artística hacia la promoción y la difusión de nuevos artistas que necesitan espacios expositivos para iniciar su carrera. Generalmente esto se hace a través de la organización de premios. En este grupo se incluyen la fundación Mainel y la Fundación Cañada-Blanch.

Esta clasificación no es excluyente, pues una fundación puede presentar como rasgo predominante de su actividad la organización de exposiciones y al mismo tiempo ofrecer visitas guiadas que completan la experiencia artística. Para esta clasificación se tomó en cuenta su actividad principal, aunque en casos como la FPAA, su programación es tan completa que su perfil se desarrolla en los cuatro ejes funcionales.

Con la información recogida hasta el momento, se observa que hay dos situaciones importantes que atender: por un lado, el mecenazgo y el apoyo al arte emergente. Se pueden encontrar propuestas que se dirigen en esta línea, pero acaban como proyectos puntuales que no dan cabida a la demanda de espacios para el arte más joven; es decir, el de las obras de reciente creación, de artistas sin recorrido reconocido. Hacen falta salas que no exijan artistas con currículums de gran trayectoria, y apuesten más por las propuestas.

Por otro lado, la evaluación es un aspecto muy importante a tener en cuenta, porque hace posible una reflexión sobre el desarrollo de los proyectos llevados a cabo. Con ella se pueden analizar los resultados y las propuestas de mejora. En 
ocasiones este aspecto se pasa por alto por diferentes motivos. Uno puede ser la falta de planificación de la evaluación como parte del proyecto, lo que hace que no dé tiempo a realizarla. Otro es que la evaluación de un proyecto cultural requiere de indicadores cuantitativos, pero también debe incluir indicadores cualitativos para analizar aspectos como la fidelidad de los visitantes, sus gustos personales y sus intereses como espectadores. Realizar una evaluación completa les exige a los profesionales tener conocimientos sobre el tema, conocer qué tipo de indicadores son los más apropiados y saber cómo interpretarlos de forma óptima.

Por último, y debido a que los recursos disponibles no siempre son suficientes y no se pueden cubrir todo tipo de actividades (expositivas, educativas, evaluadoras), sería adecuado plantear un trabajo en red colaborativo en el que se establecieran las sinergias necesarias para cubrir las necesidades que quedan pendientes en los proyectos culturales. Buscar los puntos de encuentro entre los diferentes agentes culturales de la ciudad, ya sean del sector público o del privado, sin perder de vista la autonomía que requieren el arte contemporáneo y la cultura. Estos pueden ser los puntos de partida para consolidar la oferta artística contemporánea de la ciudad.

\section{Referencias}

Aguado Quintero, L. (2010). Estadísticas culturales: una mirada desde la economía de la cultura. Cuadernos de administración, 23(41), pp. 107-141. Recuperado de: https://idus.us.es/xmlui/bitstream/handle/11441/16513/file_1.pdf?seque nce $=1$

Cabra de Luna, M. (2001). Propuesta de balance social para fundaciones. CIRIECEspaña, Revista de Economía Pública, Social y Cooperativa, núm. 39, pp. 51-78. Recuperado de: http://www.redalyc.org/articulo.oa?id=17403904

Del Alisal, E. (2007). Espacios para el arte: lugares en continua redefinición. Em Questão, 13(2), pp. 225-233. Recuperado de: http://www.redalyc.org/pdf/4656/465645957003.pdf

De la Torre, I. (2003). Los fundamentos del tercer sector. Revista Internacional de Sociología (RIS), 61(35), pp. 105-125. Recuperado de: http://revintsociologia.revistas.csic.es/index.php/revintsociologia/article/ view/302

Eco, U. (1995). Apocalípticos e integrados. Barcelona, España: Tusquets. 
Estatut d'Autonomia de la Comunitat Valenciana. Recuperado de: https://www.cortsvalencianes.es/ca-va/composicion/normas/estautonomia

Florido Arreza, A. (2011). La maldición de la Mona Lisa. El nuevo precio del arte. ASRI Arte y Sociedad, vol. 0. Recuperado de: https://dialnet.unirioja.es/servlet/articulo?codigo $=3792086$

Fontal, O. (2006). Claves del patrimonio cultural del presente y desde el presente para abordar su enseñanza. Pulso, vol. 29, pp. 9-31. Recuperado de: https://ebuah.uah.es/dspace/handle/10017/5160

Gómez Redondo, C. (2011). Procesos de patrimonialización en el arte contemporáneo. EARI-Educación Artística Revista de Investigación, vol. 2, pp. 108-112. Recuperado de: https://ojs3.uv.es/index.php/eari/article/view/2511/2060

Jiménez Escobar, J. (2013). Nuevas realidades en el Tercer sector: Las aportaciones jurídico-organizativas de las fundaciones cívicas. REVESCO, núm. 110, pp. 163-191. Recuperado de: https://dialnet.unirioja.es/servlet/articulo?codigo $=4190428$

Ley 9/2014, de 29 de diciembre, de impulso de la actividad y del mecenazgo cultural en la Comunitat Valenciana. Recuperado de: http://www.dogv.gva.es/portal/ficha_disposicion.jsp?L=1\&sig=011510\% 2F2014

Ley 20/2018, de 25 de julio, de la Generalitat, del mecenazgo cultural, científico y deportivo no profesional en la Comunitat Valenciana. [2018/7451]. Recuperado

de: https://www.dogv.gva.es/portal/ficha_disposicion_pc.jsp?sig=007299/20 18\&L=1Ley 50/2002, de 26 de diciembre, de Fundaciones, B.O.E. 310, 2013, 45504 - 45515, Spain Recuperado de: https://www.boe.es/boe/dias/2002/12/27/pdfs/A4550445515.pdfMartínez Álvarez, O. (2002). Tres elements d'alquímia: pedagogia, ciutat i museu. Revista Catalana de pedagogía, vol. 1, pp. 267291. Recuperado de http://revistes.iec.cat/index.php/RCP/article/view/3800/3799 
Orden del 6 de febrero de 1991, de la Conselleria de Cultura, Educación y Ciencia, por la que se regula el reconocimiento de museos y colecciones museográficas permanentes de la Comunidad Valenciana, D.O.G.V. núm. 1494 de 28.02.1991, 0596/1991, Comunidad Valenciana, España. Recuperado

de: http://www.dogv.gva.es/portal/ficha_disposicion_pc.jsp?sig=0596/1991\& $\mathrm{L}=1$

Palencia-Lefler Ors, M. (2007). Donación, mecenazgo y patrocinio como técnicas de relaciones públicas. Análisis, vol. 35, pp. 153-170. Recuperado de: http://eprints.rclis.org/15884/1/article\%20analisi.pdf

Raussell, P., Abeledo, R., Boix, R., De Miguel, B., Hervás, J. L., Marcos, F., Vila, L. (2012). Culture as a factor of economic and social innovation. SOSTENUTO final report.

Roselló Cerezuela, D. (2008). Diseño y evaluación de proyectos culturales. Barcelona: Ariel.

Segovia, C., Marrades, R., Rausell, P., Raül A. (2015). Spaces for Innovation, Creativity and Culture. Recuperado de: https://www.uv.es/spaces/\#public-space

Tatarkiewicz, W. (1986). Historia de seis ideas. Arte, belleza, forma, creatividad, mímesis, experiencia estética. Madrid: Tecnos.

Wolff, J. (2018). La producción social del arte. Madrid, España: Istmo. 